PROCEEDINGS OF THE

AMERICAN MATHEMATICAL SOCIETY

Volume 133, Number 1, Pages 1-10

S 0002-9939(04)07716-0

Article electronically published on August 24, 2004

\title{
JOINS OF PROJECTIVE VARIETIES AND MULTISECANT SPACES
}

\author{
E. BALLICO
}

(Communicated by Michael Stillman)

\begin{abstract}
Let $X_{1}, \ldots, X_{s} \subset \mathbf{P}^{N}, s \geq 1$, be integral varieties. For any integers $k_{i}>0,1 \leq i \leq s$, and $t \geq 0$ set $\vec{k}:=\left(k_{1}, \ldots, k_{s}\right)$ and $\vec{X}:=$ $\left(X_{1}, \ldots, X_{s}\right)$. Let $\operatorname{Sec}(\vec{X} ; t, \vec{k})$ be the set of all linear $t$-spaces contained in a linear $\left(k_{1}+\cdots+k_{s}-1\right)$-space spanned by $k_{1}$ points of $X_{1}, k_{2}$ points of $X_{2}, \ldots, k_{s}$ points of $X_{s}$. Here we study some cases where $\operatorname{Sec}(\vec{X} ; t, \vec{k})$ has the expected dimension. The case $s=1$ was recently considered by Chiantini and Coppens and we follow their ideas. The two main results of the paper consider cases where each $X_{i}$ is a surface, more particularly:

$$
s=3, k_{1}=k_{2}=k_{3}=1 \text { and } t=1
$$

or

$$
s=2, k_{1}=2, k_{2}=1 \text { and } t=1 .
$$
\end{abstract}

\section{INTRODUCTION}

L. Chiantini and M. Coppens revived a piece of classical projective geometry (see 6] and references therein): the study of the set of all linear spaces contained in the secant varieties of an integral variety $X \subset \mathbf{P}^{N}$. For further papers on this topic, see [5], 8] and 9]. Let $G(t+1, N+1)$ be the Grassmannian of all $t$-dimensional linear subspaces of $\mathbf{P}^{N}$. The order $k$ secant variety of $X$ is the join of $k$ copies of $X$. In this paper we fix $s$ varieties $X_{i} \subset \mathbf{P}^{N}, 1 \leq i \leq s$, and consider the closure in $G(t+1, N+1)$ of the set of all $t$-spaces contained in a $\left(k_{1}+\cdots+k_{s}-1\right)$-space spanned by $k_{1}$ points of $X_{1}, k_{2}$ points of $X_{2}, \ldots, k_{s}$ points of $X_{s}$. The case $s=1$ is the case considered in [6] and we will often use the ideas contained in [6].

We work over an algebraically closed field $\mathbf{K}$ with $\operatorname{char}(\mathbf{K})=0$. For any two integral varieties $X, Y$ of $\mathbf{P}^{N}$, let $[X ; Y]$ be the join of $X$ and $Y$; thus if $X=Y=$ $\{P\}$ with $P$ a point, then $[X ; Y]=\{P\}$, while in all other cases $[X ; Y]$ is the closure in $\mathbf{P}^{N}$ of the union of all lines $\langle\{A, B\}\rangle$ spanned by some $A \in X$ and some $B \in Y$ with $A \neq B$.

Fix integers $N \geq 3, s>0$ and $k_{i} \geq 0,1 \leq i \leq s$. Set $\vec{k}:=\left(k_{1}, \ldots, k_{s}\right)$ and $|\vec{k}|:=$ $k_{1}+\cdots+k_{s}-1$. Let $t$ be an integer such that $0 \leq t \leq|\vec{k}|$. Fix $s$ irreducible varieties $X_{i} \subset \mathbf{P}^{N}, 1 \leq i \leq s$. Usually, we will be interested in the case $X_{i} \neq X_{j}$ for $i \neq j$, since the general case may be reduced to this case by decreasing $s$, but with the same

Received by the editors August 16, 2002.

2000 Mathematics Subject Classification. Primary 14N05, 14M15.

Key words and phrases. Joins, multisecant spaces, secant variety, Grassmannian.

The author was partially supported by MIUR and GNSAGA of INdAM (Italy).

(C)2004 American Mathematical Society 
value of $|\vec{k}|$. The $(t, \vec{k})$-secant variety $\operatorname{Sec}(\vec{X} ; t, \vec{k})$ of $\vec{X}$ is the closure in $G(t+1, N+1)$ of all $t$-spaces contained in a $|\vec{k}|$-dimensional linear subspace of $\mathbf{P}^{N}$ spanned by $k_{1}$ points of $X_{1}, k_{2}$ points of $X_{2}, \ldots, k_{s}$ points of $X_{s}$. Set $\operatorname{Sec}(\vec{X} ; \vec{k}):=\operatorname{Sec}(\vec{X} ; 0, \vec{k}) \subseteq$ $\mathbf{P}^{N}$. The $(0, \vec{k})$-secant variety $\operatorname{Sec}(\vec{X} ; \vec{k})$ of $\vec{X}$ will be called the $\vec{k}$-secant variety of $\vec{X}$. Set $n_{i}:=\operatorname{dim}\left(X_{i}\right)$. We have $\operatorname{dim}(\operatorname{Sec}(\vec{X} ; \vec{k})) \leq \min \left\{N, \sum_{i=1}^{s} k_{i}\left(n_{i}+1\right)-1\right\}$ and $\operatorname{dim}(\operatorname{Sec}(\vec{X} ; t, \vec{k})) \leq \min \left\{(t+1)(N-t), \sum_{i=1}^{s} k_{i} n_{i}+(|\vec{k}|-t)(t+1)\right\}$. We will say that $\vec{X}$ is $\vec{k}$-defective (resp. $(t, \vec{k})$-defective) if

$$
\operatorname{dim}(\operatorname{Sec}(\vec{X} ; \vec{k}))<\min \left\{N, \sum_{i=1}^{s} k_{i}\left(n_{i}+1\right)-1\right\}
$$

$\left(\right.$ resp. $\left.\operatorname{dim}(\operatorname{Sec}(\vec{X} ; t, \vec{k}))<\min \left\{(t+1)(N-t), \sum_{i=1}^{s} k_{i} n_{i}+(|\vec{k}|-t)(t+1)\right\}\right)$. If $\vec{X}$ is $\vec{k}$-defective (resp. $(t, \vec{k})$-defective) the integer

$$
\delta(\vec{X} ; \vec{k}):=\sum_{i=1}^{s} k_{i}\left(n_{i}+1\right)-1-\operatorname{dim}(\operatorname{Sec}(\vec{X} ; \vec{k}))
$$

$\left(\operatorname{resp} . \delta(\vec{X} ; t, \vec{k}):=\sum_{i=1}^{s} k_{i} n_{i}+(|\vec{k}|-t)(t+1)-\operatorname{dim}(\operatorname{Sec}(\vec{X} ; t, \vec{k}))\right)$ will be called the total order of $\vec{k}$-defectivity (resp. $(t, \vec{k})$-defectivity) of $\vec{X}$.

It seems very natural to start the study of $(t, \vec{k})$-defectivity from the case $\operatorname{dim}\left(X_{i}\right)$ $=1$ for every $i$. For the case in which each $X_{i}$ is a non-degenerate curve, see Corollary 1 and Theorem 4 in section 3 . For a more general defectivity result for non-degenerate curves, see [3]; in the quoted paper we considered the set of all flags of linear spaces contained in a $|\vec{k}|$-dimensional linear space instead of the set of all $t$-dimensional linear spaces. Obviously, the interested reader may do other related cases (e.g. some degenerate curves or a surface and $s-1$ non-degenerate curves). We stress that degenerate varieties may not give defective $s$-ples (e.g. when $k_{i}=1$ for all $i$ take as $X_{i}, 1 \leq i \leq s$, linearly independent linear subspaces). For degenerate varieties, see also Remark 3 We believe that when $s \geq 2$, the mutual position of the varieties is more important than their structure. For examples of defectivity when one of the varieties is a cone, see Remark 1 For a complete analysis of a toy case, see Example 1. We raise the following question.

Question 1. Fix integers $s \geq 2, k_{i}>0,1 \leq i \leq s, t>0$, and integral varieties $X_{i} \subset \mathbf{P}^{N}, 1 \leq i \leq s$. Set $\vec{X}:=\left(X_{1}, \ldots, X_{s}\right), \vec{k}:=\left(k_{1}, \ldots, k_{s}\right), \vec{Y}:=\left(X_{1}, \ldots, X_{s-1}\right)$ and $\vec{m}:=\left(k_{1}, \ldots, k_{s-1}\right)$. Assume that $\operatorname{Sec}(\vec{Y} ; t, \vec{m})$ has the expected dimension and that $X_{s}$ is a curve. Are there reasonable conditions on $X_{s}$ assuring that $\operatorname{Sec}(\vec{X} ; t, \vec{k})$ has the expected dimension? More generally, for any $\vec{Y}$ has $\operatorname{Sec}(\vec{X} ; t, \vec{k})$ the maximal possible dimension compatible with the dimension of $\operatorname{Sec}(\vec{Y} ; t, \vec{m})$ ?

It is well known and easy to show that in the case $t=0$ a sufficient condition for an affirmative answer to Question 1 is that $X_{s}$ is a non-degenerate curve (see Corollary 1 and Remark 3 for more precise results). See Theorem 4 for the case $t=1$ and [3] when $\operatorname{dim}\left(X_{i}\right)=1$ and each $X_{i}$ is non-degenerate.

Our main results are non-existence results for the $(1, \vec{k})$-defectivity of joins of surfaces. In section 2 we will prove the following results.

Theorem 1. Let $X_{1}, X_{2}$ and $X_{3}$ be integral non-degenerate surfaces of $\mathbf{P}^{N}, N \geq 5$, such that $X_{i} \neq X_{j}$ for $i \neq j$. Assume $\operatorname{dim}\left(\left[X_{1} ; X_{2}\right]\right)=5$ and that $X_{3}$ is not 
a cone. Set $\vec{k}=(1,1,1)$. Then $\vec{X}:=\left(X_{1}, X_{2}, X_{3}\right)$ is not $(1, \vec{k})$-defective, i.e. $\operatorname{dim}(\operatorname{Sec}(\vec{X} ; 1, \vec{k}))=8$.

Theorem 2. Let $X_{1}$ and $X_{2}$ be integral non-degenerate surfaces of $\mathbf{P}^{N}, N \geq 5$, such that $X_{1} \neq X_{2}$. Assume that neither $X_{1}$ nor $X_{2}$ is a cone. Set $\vec{k}:=(2,1)$. Then $\vec{X}:=\left(X_{1}, X_{2}\right)$ is not $(1, \vec{k})$-defective.

The condition $\operatorname{dim}\left(\left[X_{1} ; X_{2}\right]\right)=5$ in the statement of Theorem 1 is very mild (see 1]). It implies that neither $X_{1}$ nor $X_{2}$ is a cone. We do not know if the condition that no $X_{i}$ is a cone is always necessary (see Remark 1), but certainly $X_{1}, X_{2}$ and $X_{3}$ cannot be cones with the same vertex (see Example 1). We do not have any construction (except cones) to obtain defective $s$-ples.

In section 3 we will give two general results on the $(t, \vec{k})$-defectivity of varieties of arbitrary dimension: an easy extension of 8 to the case of joins of different varieties (Theorem 3) and a non-defectivity result with respect to lines (Theorem 4).

\section{Proofs of Theorems 1 and 2}

In this section we will prove Theorems 1 and 2 and give the toy example and the remark on cones promised in the introduction.

For any subset $S$ of $\mathbf{P}^{N}$, let $\langle S\rangle$ be its linear span. We start with a baby example.

Example 1. Fix integers $s \geq 2, n_{i} \geq 2,1 \leq i \leq s, k_{i}>0,1 \leq i \leq s, N>$ $\min _{1 \leq i \leq s}\left\{n_{i}\right\}$ and $t$ such that $0 \leq t \leq k_{1}+\cdots+k_{s}-1$. Fix $P \in \mathbf{P}^{N}$ and non-degenerate varieties $X_{i} \subset \mathbf{P}^{N}, 1 \leq i \leq s$, such that $\operatorname{dim}\left(X_{i}\right)=n_{i}$. Set $\vec{X}:=\left(X_{1}, \ldots, X_{s}\right)$ and $\vec{k}:=\left(k_{1}, \ldots, k_{s}\right)$. Assume that each $X_{i}$ is a cone with vertex containing $P$. For all hyperplanes $H, M$ of $\mathbf{P}^{N}$ such that $P \notin H \cup M$ the $s$-ples $\left(X_{1} \cap H, \ldots, X_{s} \cap H\right)$ and $\left(X_{1} \cap M, \ldots, X_{s} \cap M\right)$ (respectively seen as $s$ ples in $H$ and in $M$ ) are projectively equivalent (use the linear projection from $P)$. Fix $H$ and set $Y_{i}:=X_{i} \cap H$ and $\vec{Y}:=\left(Y_{1}, \ldots, Y_{s}\right)$. Fix $S_{i} \subset Y_{i}, 1 \leq i \leq s$, such that $\operatorname{card}\left(S_{i}\right):=k_{i}$ and $\operatorname{dim}\left(\left\langle S_{1} \cup \cdots \cup S_{s}\right\rangle\right)=k_{1}+\cdots+k_{s}-1$. Thus $\operatorname{dim}\left(\left\langle S_{1} \cup \cdots \cup S_{s} \cup\{P\}\right\rangle\right)=k_{1}+\cdots+k_{s}$, and for every $t$-dimensional linear space $D \subset\left\langle S_{1} \cup \cdots \cup S_{s}\right\rangle$ the $(t+1)$-dimensional linear space $[D ;\{P\}]$ contains a $(t+1)$ dimensional family of $t$-dimensional linear spaces not containing $P$ and mapped isomorphically onto $D$ by the linear projection from $P$. Fix any such $t$-dimensional linear space $D^{\prime}$. There is a hyperplane $M$ of $\mathbf{P}^{N}$ such that $D^{\prime} \subset M$ and $P \notin M$. Set

$$
S_{i}^{M}:=\bigcup_{Q \in S_{i}}\langle\{Q, P\}\rangle \cap M \subset X_{i} .
$$

Thus $\operatorname{dim}\left(\left\langle S_{1}^{M} \cup \cdots \cup S_{s}^{M}\right\rangle\right)=k_{1}+\cdots+k_{s}-1, D^{\prime} \subset\left\langle S_{1}^{M} \cup \cdots \cup S_{s}^{M}\right\rangle$ and hence $D^{\prime} \in \operatorname{Sec}(\vec{X} ; t, \vec{k})$. Thus if $\operatorname{Sec}(\vec{Y} ; t, \vec{k})=G(t+1, N)$, then

$$
\operatorname{Sec}(\vec{X} ; t, \vec{k})=G(t+1, N+1),
$$

while if $\operatorname{Sec}(\vec{Y} ; t, \vec{k}) \neq G(t+1, N)$, then $\delta(\vec{X} ; t, \vec{k})=\delta(\vec{Y} ; t, \vec{k})+k_{1}+\cdots+k_{s}-1-t$. Thus in the former case $(\vec{X}, \vec{k})$ is not $(t, \vec{k})$-defective. In the latter case if $t<k_{1}+$ $\cdots+k_{s}-1$, then $\vec{X}$ is $(t, \vec{k})$-defective. The fact that in the definition of defectivity we have to take the cut-off function min implies that very natural constructions do not always (but only almost always) give degenerate $s$-ples. 
Remark 1. Fix $P \in \mathbf{P}^{N}$ and also fix a locally closed and irreducible subset $T$ of $G(t+1, N+1)$ such that $P \notin A$ for every $A \in T$. Let $T * P$ be the closure in $G(t+1, N+1)$ of the set of all $B \in G(t+1, N+1)$ contained in some $(t+1)$ dimensional linear space $\langle A \cup\{P\}\rangle$ for some $A \in T$. Fix integers $s \geq 1, k_{i}>0$, $1 \leq i \leq s$, and integral varieties $X_{i} \subset \mathbf{P}^{N}, 1 \leq i \leq s$. Assume that $X_{s}$ is a positive-dimensional cone with vertex containing $P \in \mathbf{P}^{N}$, say $X_{s}=[D ;\{P\}]$ with $\operatorname{dim}(D)=\operatorname{dim}\left(X_{s}\right)-1$ and $P \notin D$. Set $\vec{X}:=\left(X_{1}, \ldots X_{s-1}, X_{s}\right)$ and $\vec{Y}:=$ $\left(X_{1}, \ldots, X_{s-1}, D\right)$. Obviously, $\operatorname{Sec}(\vec{X} ; \vec{k})$ is the cone with $P$ as vertex and $\operatorname{Sec}(\vec{Y} ; \vec{k})$ as a basis. Hence $\vec{X}$ is $\vec{k}$-defective if $k_{s} \geq 2$ and $\operatorname{dim}(\operatorname{Sec}(\vec{Y} ; \vec{k})) \leq N-2$. Now assume $t>0$. Let $\operatorname{Sec}(\vec{Y} ; t, \vec{k})^{\prime}$ be the open subset of $\operatorname{Sec}(\vec{Y} ; t, \vec{k})$ formed by the $t$-planes not containing $P$. It is easy to check that $\operatorname{Sec}(\vec{X} ; t, \vec{k})=\operatorname{Sec}(\vec{Y} ; t, \vec{k})^{\prime} * P$. Since $\operatorname{dim}(G(t+1, t+2))=t+1$, we obtain $\operatorname{dim}(\operatorname{Sec}(\vec{X} ; t, \vec{k})) \leq \operatorname{dim}(\operatorname{Sec}(\vec{Y} ; t, \vec{k}))+$ $t+1$. Hence $\operatorname{Sec}(\vec{X} ; t, \vec{k})$ is $(t, \vec{k})$-defective if $k_{s} \geq t+2$ and $\operatorname{dim}(\operatorname{Sec}(\vec{Y} ; t, \vec{k}))+t+1<$ $(t+1)(N-t)$.

Remark 2. Let $X_{i} \subset \mathbf{P}^{m}, 1 \leq i \leq 3, m \geq 3$, be integral surfaces such that $\left\langle X_{1} \cup X_{2} \cup X_{3}\right\rangle=\mathbf{P}^{m}$ and either $X_{1} \neq X_{2}$ or $X_{1}$ is not a plane. It is easy to check that for a general $\left(A_{1}, A_{2}, A_{3}\right) \in X_{1} \times X_{2} \times X_{3}$ and a general $\left(B_{1}, B_{2}, B_{3}, B_{4}\right) \in X_{1} \times$ $X_{2} \times X_{3} \times X_{4}$ we have $\operatorname{dim}\left(\left\langle\left\{A_{1}, A_{2}, A_{3}\right\}\right\rangle\right)=2$ and $\operatorname{dim}\left(\left\langle\left\{B_{1}, B_{2}, B_{3}, B_{4}\right\}\right\rangle\right)=3$.

Lemma 1. Let $X_{i} \subset \mathbf{P}^{4}, 1 \leq i \leq 2$, be integral non-degenerate surfaces. Then for a general $\left(A_{1}, A_{2}\right) \in X_{1} \times X_{2}$ we have $\left\langle\left\{A_{1}, A_{2}\right\}\right\rangle \cap\left(X_{1} \cup X_{2}\right)=\left\{A_{1}, A_{2}\right\}$.

Proof. If $X_{1}=X_{2}$, then the lemma is [6], Cor. 1.3, for the invariants $r=4, n=2$ and $k=1$. Assume $X_{1} \neq X_{2}$. First assume that $\left\langle\left\{A_{1}, A_{2}\right\}\right\rangle \cap\left(X_{1} \cup X_{2}\right)$ contains $B \in X_{1} \backslash\left\{A_{1}\right\}$. Then for a general $A_{2} \in X_{2}$ the restriction to $X_{1}$ of the linear projection $\mathbf{P}^{4} \backslash\left\{A_{2}\right\} \rightarrow \mathbf{P}^{3}$ from $A_{2}$ is not birational. Since $X_{2} \neq X_{1}$, this implies that $X_{2}$ is contained in the so-called Segre locus $\Sigma\left(X_{1}\right)$ of $X_{1}$, contradicting the inequality $\operatorname{dim}\left(\Sigma\left(X_{1}\right)\right) \leq 1$ proved in [4, Th. 1. Similarly, if $\left\langle\left\{A_{1}, A_{2}\right\}\right\rangle \cap\left(X_{1} \cup X_{2}\right)$ contains $B \in X_{2} \backslash\left\{A_{2}\right\}$, we see that $X_{1}$ is contained in the Segre locus of $X_{2}$, contradicting [4], Th. 1 .

Lemma 2. Let $X_{i} \subset \mathbf{P}^{5}, 1 \leq i \leq 3$, be integral surfaces such that $X_{i} \neq X_{j}$ and $\left\langle X_{i} \cup X_{j}\right\rangle=\mathbf{P}^{5}$ for all $i, j \in\{1,2,3\}$ with $i \neq j$. Then for a general $\left(A_{1}, A_{2}, A_{3}\right) \in$ $X_{1} \times X_{2} \times X_{3}$ we have $\left\langle\left\{A_{1}, A_{2}, A_{3}\right\}\right\rangle \cap\left(X_{1} \cup X_{2} \cup X_{3}\right)=\left\{A_{1}, A_{2}, A_{3}\right\}$.

Proof. Assume that for a general $\left(A_{1}, A_{2}, A_{3}\right) \in X_{1} \times X_{2} \times X_{3}$ we have $\left\langle\left\{A_{1}, A_{2}, A_{3}\right\}\right\rangle$ $\cap\left(X_{1} \cup X_{2} \cup X_{3}\right) \neq\left\{A_{1}, A_{2}, A_{3}\right\}$. Just to fix the notation assume that $\left\langle\left\{A_{1}, A_{2}, A_{3}\right\}\right\rangle$ $\cap\left(X_{1} \cup X_{2} \cup X_{3}\right)$ contains $B \in X_{1} \backslash\left\{A_{3}\right\}$. Let $f: \mathbf{P}^{5} \backslash\left\{A_{3}\right\} \rightarrow \mathbf{P}^{4}$ be the linear projection from the point $A_{3}$ and $g: \mathbf{P}^{4} \backslash\left\{f\left(A_{2}\right)\right\} \rightarrow \mathbf{P}^{3}$ the linear projection from the point $f\left(A_{2}\right)$. Using that $X_{3} \neq X_{2}, X_{3} \neq X_{1}, X_{3}$ is not contained in $\left\langle X_{i}\right\rangle$ $(i=1,2)$ if $X_{i}$ is degenerate and $A_{3}$ is general in $X_{3}$, we obtain $A_{3} \notin X_{1} \cup X_{2}$, $\left\langle f\left(X_{1}\right) \cup f\left(X_{2}\right)\right\rangle=\mathbf{P}^{4}$ and $\operatorname{dim}\left(\left\langle f\left(X_{i}\right)\right\rangle=\min \left\{4, \operatorname{dim}\left(\left\langle X_{i}\right\rangle\right)\right\}\right.$ for $i=1,2$. Hence either $\left\langle f\left(X_{1}\right)\right\rangle=\mathbf{P}^{4}$ or $f\left(X_{2}\right) \nsubseteq\left\langle f\left(X_{1}\right)\right\rangle$. If $\left\langle f\left(X_{1}\right)\right\rangle \neq \mathbf{P}^{4}$, by the generality of $f\left(A_{2}\right)$ in $f\left(X_{2}\right)$ we obtain that $g \mid f\left(X_{1}\right)$ is injective (here we just take any $f\left(A_{2}\right) \notin$ $\left\langle f\left(X_{1}\right)\right\rangle$ ), contradicting the existence of $A_{1}$ and $B$ (even if $A_{1}$ is not assumed to be general in $\left.X_{1}\right)$ such that $A_{1} \neq B$ and $B \in\left\langle\left\{A_{1}, A_{2}, A_{3}\right\}\right\rangle \cap\left(X_{1} \cup X_{2} \cup X_{3}\right)$. Hence we may assume $\left\langle f\left(X_{1}\right)\right\rangle=\mathbf{P}^{4}$. To obtain a contradiction it is sufficient to show that $g \mid f\left(X_{1}\right)$ is birational. Assume that $g \mid f\left(X_{1}\right)$ is not birational. Since $f\left(A_{2}\right)$ is a general point of $f\left(X_{2}\right)$ and $f\left(X_{1}\right) \neq f\left(X_{2}\right)$, we obtain that a general point of $f\left(X_{2}\right) \backslash f\left(X_{1}\right)$ is in the Segre locus $\Sigma\left(f\left(X_{1}\right)\right)$ of $f\left(X_{1}\right)$, contradicting [4], Th. 1. 
Lemma 3. Let $X_{i} \subset \mathbf{P}^{5}, 1 \leq i \leq 2$, be integral surfaces such that $X_{1} \neq X_{2}$, $\left\langle X_{1} \cup X_{2}\right\rangle=\mathbf{P}^{5}$ and $\operatorname{dim}\left(\left\langle X_{1}\right\rangle\right) \geq 4$. Then for a general $\left(A_{1}, A_{2}, B\right) \in X_{1} \times X_{1} \times X_{2}$ we have $\left\langle\left\{A_{1}, A_{2}, B\right\}\right\rangle \cap\left(X_{1} \cup X_{2}\right)=\left\{A_{1}, A_{2}, A_{3}\right\}$.

Proof. Assume that for a general $\left(A_{1}, A_{2}, B\right) \in X_{1} \times X_{1} \times X_{2}$ we have $\left\langle\left\{A_{1}, A_{2}, B\right\}\right\rangle$ $\cap\left(X_{1} \cup X_{2}\right) \neq\left\{A_{1}, A_{2}, B\right\}$. Let $f: \mathbf{P}^{5} \backslash\{B\} \rightarrow \mathbf{P}^{4}$ be the linear projection from $B$. First assume that $\left\langle\left\{A_{1}, A_{2}, B\right\}\right\rangle \cap\left(X_{1} \cup X_{2}\right)$ contains $D \in X_{1} \backslash\left\{A_{1}, A_{2}\right\}$. Since $B$ is general in $X_{2}, f\left(X_{1}\right)$ spans $\mathbf{P}^{4}$. Hence a general secant line of $f\left(X_{1}\right)$ is not a trisecant line ([6], Cor. 1.3). Since $f(D) \in\left\langle f\left(A_{1}\right), f\left(A_{2}\right)\right\rangle$, we obtain that either $f(D)=f\left(A_{1}\right)$ or $f(D)=f\left(A_{2}\right)$. Just to fix the notation we assume $f(D)=f\left(A_{1}\right)$. Since $A_{1}$ is general in $X_{1}$, we obtain that $f \mid X_{1}$ is not birational. Hence a general $B \in X_{2}$ is in the Segre locus $\Sigma\left(X_{1}\right)$ of $X_{1}$, contradicting [4, Th. 1. Now assume that $\left\langle\left\{A_{1}, A_{2}, B\right\}\right\rangle \cap\left(X_{1} \cup X_{2}\right)$ contains $C \in X_{2} \backslash\{B\}$. We obtain that any secant line to $f\left(X_{1}\right)$ intersects $f\left(X_{2} \backslash\{B\}\right)$. Hence $f\left(X_{2}\right) \subseteq \Sigma\left(f\left(X_{1}\right)\right)$, a contradiction.

Lemma 4. Let $C, D \subset \mathbf{P}^{m}, m \geq 3$, be integral non-degenerate curves. Assume $C \neq D$. Then a general secant line to $C$ is not secant to $D$.

Proof. Assume that this is not true and fix a general $P \in C$. By assumption for a general $Q \in C$ the line $\langle\{P, Q\}\rangle$ is secant to $D$. Hence the linear projection from $P$ is not birational. Thus a general $P \in C$ is contained in the Segre locus $\Sigma(D)$ of $D$, contradicting [4, Th. 1 .

Lemma 5. Let $X_{i} \subset \mathbf{P}^{5}, 1 \leq i \leq 2$, be integral surfaces such that $\left\langle X_{1} \cup X_{2}\right\rangle=\mathbf{P}^{5}$ and neither $X_{1}$ nor $X_{2}$ is a plane. Then for a general $\left(A_{1}, A_{2}, B_{1}, B_{2}\right) \in X_{1} \times X_{1} \times$ $X_{2} \times X_{2}$ the set $\left\langle\left\{A_{1}, A_{2}, B_{1}, B_{2}\right\}\right\rangle \cap\left(X_{1} \cup X_{2}\right)$ is finite.

Proof. Assume that the lemma is false and that for instance $\left\langle\left\{A_{1}, A_{2}, B_{1}, B_{2}\right\}\right\rangle \cap$ $\left(X_{1} \cup X_{2}\right)$ contains an integral curve $C \subset X_{2}$. Let $f: \mathbf{P}^{5} \backslash\left\langle\left\{A_{1}, A_{2}\right\}\right\rangle \rightarrow \mathbf{P}^{3}$ be the linear projection from the line $\left\langle\left\{A_{1}, A_{2}\right\}\right\rangle$. First assume that $C$ is not contained in a plane containing $\left\langle\left\{A_{1}, A_{2}\right\}\right\rangle$. Thus $f\left(C \backslash C \cap\left\langle\left\{A_{1}, A_{2}\right\}\right\rangle\right)$ is a curve. Since $f\left(C \backslash C \cap\left\langle\left\{A_{1}, A_{2}\right\}\right\rangle\right)$ is contained in the line $\left\langle\left\{f\left(B_{1}\right), f\left(B_{2}\right)\right\}\right\rangle$, we obtain $\left\langle\left\{f\left(B_{1}\right), f\left(B_{2}\right)\right\}\right\rangle \subseteq f\left(X_{2} \backslash X_{2} \cap\left\langle\left\{A_{1}, A_{2}\right\}\right\rangle\right)$. Since $\left(f\left(B_{1}\right), f\left(B_{2}\right)\right)$ is general in $f\left(X_{2}\right) \times f\left(X_{2}\right), f\left(X_{2}\right)$ is a plane. Thus $\operatorname{dim}\left(\left\langle X_{2} \cup\left\{A_{1}, A_{2}\right\}\right\rangle\right)=4$. By the generality of $A_{1}$ and $A_{2}$ and the assumption $\left\langle X_{1} \cup X_{2}\right\rangle=\mathbf{P}^{5}$, we obtain that $X_{2}$ is a plane, a contradiction. Now assume that $C$ is contained in a plane $M$ containing $\left\langle\left\{A_{1}, A_{2}\right\}\right\rangle$. Varying $A_{1}$ and $A_{2}$ we obtain that $X_{2}$ contains at least a two-dimensional family of plane curves. If $\operatorname{dim}\left(\left\langle X_{2}\right\rangle\right) \geq 4$, we obtain that $C$ is a plane conic and $X_{2}$ is either the Veronese surface or a projection of the Veronese surface ([6], Segre's lemma at p. 623). We have $M=\langle C\rangle,\left\{A_{1}, A_{2}\right\} \subset M$ and the scheme-theoretic intersection of $\left\langle\left\{A_{1}, A_{2}\right\}\right\rangle$ with $C$ has length two. Hence any secant line to $X_{1}$ is secant to $X_{2}$. Take a general hyperplane $H$ of $\mathbf{P}^{5}$ and apply Lemma 4 to a general projection of the curves $X_{1} \cap H$ and $X_{2} \cap H$ in $\mathbf{P}^{3}$ to obtain a contradiction. Now assume $\operatorname{dim}\left(\left\langle X_{2}\right\rangle\right) \leq$ 3. Hence $\operatorname{dim}\left(\left\langle X_{2}\right\rangle\right)=3$. Since $\left\langle X_{1} \cup X_{2}\right\rangle=\mathbf{P}^{5}$, for general $\left(A_{1}, A_{2}\right) \in X_{1} \times X_{1}$ we have $\left\langle\left\{A_{1}, A_{2}\right\}\right\rangle \cap\left\langle X_{2}\right\rangle=\emptyset$, and hence $\left\langle\left\{A_{1}, A_{2}, B_{1}, B_{2}\right\}\right\rangle \cap X_{2}$ is contained in $\left\langle\left\{B_{1}, B_{2}\right\}\right\rangle \cap X_{2}$ and hence it is finite, a contradiction.

Look at the set-up of Lemma 5 . If $X_{2}$ is a plane, then $\left\langle\left\{A_{1}, A_{2}, B_{1}, B_{2}\right\}\right\rangle \cap X_{2}$ is a line.

Proof of Theorem 1. We divide the proof into 9 steps. Steps 1 to 7 are just the translation in our set-up of the corresponding steps in the proof of the Theorem in 
section 2 of [6]. The degree 3 curve arising in Step 10 of [6] does not appear in our proof of Theorem 1 because the integer 3 is now distributed between $X_{1}, X_{2}$ and $X_{3}$. Instead, in our proof of Theorem 1 we obtain a one-dimensional family $\Phi$ of lines contained in $X_{3}$. Furthermore, in Step 9 we will use again that $X_{2} \neq X_{3}$. Therefore the proof of Theorem 1 is shorter and easier than the proof of the Theorem in [6], $\S 2$.

Step 1. Taking a general linear projection into $\mathbf{P}^{5}$ we reduce to the case $N=5$. By assumption we have $\operatorname{dim}\left(\left[X_{1} ; X_{2}\right]\right)=5$, i.e. $\left[X_{1} ; X_{2}\right]=\mathbf{P}^{5}$. Let $J:=\{(\Pi, Q)$ : $Q \in \Pi\} \subset \operatorname{Sec}(\vec{X} ; \vec{k}) \times \mathbf{P}^{5}$ be the incidence variety and $q: J \rightarrow \operatorname{Sec}(\vec{X} ; \vec{k}), p: J \rightarrow$ $\mathbf{P}^{5}$ the projections. We have $\operatorname{dim}(J)=8$ (see e.g. the proof of [6], Prop. 1.1). Since $\left[X_{1} ; X_{2}\right]=\mathbf{P}^{5}, p$ is surjective. Thus for a general $P \in \mathbf{P}^{5}$ every irreducible component of $p^{-1}(P)$ has dimension 3. Fix a general $P \in \mathbf{P}^{5}$ and choose one irreducible component $L_{P}$ of $p^{-1}(P)$.

Step 2. Let $W_{P}:=p\left(q^{-1}\left(q\left(L_{P}\right)\right)\right)$ be the union of all planes belonging to $L_{P}$. In this step we will check the existence of a choice of the component $L_{P}$ of $p^{-1}(P)$ such that $W_{P}$ is an irreducible variety containing $X_{3} . W_{P}$ is irreducible because $L_{P}$ is irreducible and $q$ is equidimensional and with irreducible fibers. Since $\left[X_{1} ; X_{2}\right]=\mathbf{P}^{5}$ and $P$ is general, there are $A \in X_{1}$ and $B \in X_{2}$ such that $P \in\langle\{A, B\}\rangle$. Hence for a general $Q \in X_{3}$ the plane $\langle\{A, B, Q\}\rangle$ belongs to $\operatorname{Sec}(\vec{X} ; \vec{k})$ and contains $P$, i.e. $\langle\{A, B, Q\}\rangle \in p^{-1}(P)$. Thus $X_{3}$ is contained in $p\left(q^{-1}\left(q\left(p^{-1}(P)\right)\right)\right)$. Since $X_{3}$ is irreducible, there is at least one irreducible component $L_{P}$ of $p^{-1}(P)$ such that $X_{3} \subseteq p\left(q^{-1}\left(q\left(L_{P}\right)\right)\right)$.

Step 3. In order to obtain a contradiction, from now on we assume that $\vec{X}$ is $(1, \vec{k})$-defective. Here we will check that $\operatorname{dim}\left(W_{P}\right)=4$. Assume $\operatorname{dim}\left(W_{P}\right)=5$. Then for a general $Q \in \mathbf{P}^{5}$ there is $\Pi \in L_{P}$ such that $Q \in \Pi$. Thus the line $\langle\{P, Q\}\rangle$ is contained in $W_{P}$. By the generality of $P$ and $Q$ we obtain $G(2,6)=\operatorname{Sec}(\vec{X} ; 1, \vec{k})$, a contradiction. Now assume $\operatorname{dim}\left(W_{P}\right) \leq 3$. Since $W_{P}$ is irreducible and contains $X_{3}$ (Step 2) and $P \notin X_{3}, \operatorname{dim}\left(W_{P}\right)=3$. For a general $Q \in X_{3}$ there is $\Pi \in W_{P}$ such that $Q \in \Pi$. Thus $\langle\{P, Q\}\rangle \subset \Pi$. Hence $W_{P}$ is the cone $\left[X_{3} ;\{P\}\right]$. Since $W_{P}$ contains a 3-dimensional family of planes, the projection of $X_{3}$ from $P$ is a surface $Y$ containing a 3-dimensional family of lines. No such surface $Y$ exists because any two general points of it would be contained in a line contained in $Y$; hence $Y$ would be a plane, while a plane does not contain a 3 -dimensional family of lines.

Step 4. Choose $A \in X_{1}$ and $B \in X_{2}$ such that $P \in\langle\{A, B\}\rangle$. Since $P$ is general, the pair $(A, B)$ is general in $X_{1} \times X_{2}$. From now on we fix a general $(A, B) \in X_{1} \times X_{2}$ and a general $P \in\langle\{A, B\}\rangle$. Let $\Psi$ be the rational map from $X_{3}$ into $G(3,6)$ that sends a general $C \in X_{3}$ into the plane $\langle\{A, B, C\}\rangle \in G(3,6)$. Call $L_{P}^{\prime}$ the closure of $\operatorname{Im}(\Psi)$. Clearly, $L_{P}^{\prime}$ is irreducible and by construction it lies in $q\left(p^{-1}(P)\right)$. We choose as $L_{P}$ a component of $q\left(p^{-1}(P)\right)$ containing $L_{P}^{\prime}$.

First Claim: We have $\operatorname{dim}\left(L_{P}^{\prime}\right)=2, p\left(q^{-1}\left(L_{P}^{\prime}\right)\right)=W_{P}=\left[\{A\} ;\left[\{B\} ; X_{3}\right]\right]$. With this choice of $L_{P}$ we have $X_{3} \subseteq W_{P}$, i.e., the statement of Step 2 holds for this component of $q\left(p^{-1}(P)\right)$.

Proof of the First Claim It is easy to check (see Lemma 2 or Lemma 5 for stronger statements) that $\Psi$ has finite fibers. Hence $\operatorname{dim}\left(L_{P}^{\prime}\right)=2$. Since $L_{P}^{\prime} \subseteq L_{P}$, we have $p\left(q^{-1}\left(L_{P}^{\prime}\right)\right) \subseteq W_{P}$. By the very definition of the rational map $\Psi$ we have $p\left(q^{-1}\left(L_{P}^{\prime}\right)\right)=\left[\{A\} ;\left[\{B\} ; X_{3}\right]\right]$. Hence to prove the First Claim it is sufficient to prove that the cone $\left[\{A\} ;\left[\{B\} ; X_{3}\right]\right]$ has dimension 4 , i.e. that $X_{3}$ is not a cone with vertex containing $B$ and that the vertex of the cone $\left[\{B\} ; X_{3}\right]$ does not contain 
$A$. Since $X_{1}$ and $X_{2}$ are non-degenerate and the pair $(A, B)$ is general in $X_{1} \times X_{2}$, both assertions are obvious.

Step 5. For any $\Pi \in L_{P} \backslash L_{P}^{\prime}$ write $\Lambda_{\Pi}$ for the linear span of $\Pi$ and the line $\langle\{A, B\}\rangle$.

Second Claim: $\Lambda_{\Pi}$ is a 3 -dimensional linear space contained in $W_{P}$, and $W_{P}$ is the closure of the union of the spaces $\Lambda_{\Pi}$ as $\Pi$ varies in $L_{P} \backslash L_{P}^{\prime}$. For a general $\Pi \in L_{P}$ the scheme $\Lambda_{\Pi} \cap X_{3}$ contains a curve.

Proof of the Second Claim Since $P \in\langle\{A, B\}\rangle \cap \Pi$ and $\Pi \notin L_{P}^{\prime}$, we have $\operatorname{dim}\left(\Lambda_{\Pi}\right)=3$. By the First Claim for a general $\Pi \in L_{P}$ and a general $Q \in \Pi$ there is $C \in X_{3}$ such that $Q \in\langle\{A, B, C\}\rangle$. Thus $\langle\{A, B, Q\}\rangle \subseteq W_{P}$ and hence $\Lambda_{\Pi} \subseteq W_{P}$. Since $\Lambda_{\Pi} \neq \Lambda_{\Pi^{\prime}}$ for a general pair $\left(\Pi, \Pi^{\prime}\right) \in L_{p} \times L_{P}$ and $\operatorname{dim}\left(W_{P}\right)=4$, $W_{P}$ is the closure of the union of the spaces $\Lambda_{\Pi}$ as $\Pi$ varies in $L_{P} \backslash L_{P}^{\prime}$. Since $X_{3} \subset W_{P}$, for a general $\Pi$ the set $\Lambda_{\Pi} \cap X_{3}$ contains a curve, proving the Second Claim.

Step 6. Here we will check that $\Lambda_{\Pi} \cap \Lambda_{\Pi^{\prime}}=\langle\{A, B\}\rangle$. Assume on the contrary that $\Lambda_{\Pi} \cap \Lambda_{\Pi^{\prime}}$ is a plane, $V$. By the Linear Lemma in [6], $\S 1$, this implies that either all 3-spaces $\Lambda_{\Pi}$ are contained in a 4-dimensional linear space $M$ or for every $R \in L_{P} \backslash L_{P}^{\prime}$ the 3 -space $\Lambda_{R}$ contains $V$. The first possibility cannot occur because $X_{3}$ is non-degenerate and contained in $W_{P}$ (Step 2) and $W_{P}$ is the closure of the union all $\Lambda_{R}$ (First Claim). Assume that for every $R \in L_{P} \backslash L_{P}^{\prime}$ the 3 -space $\Lambda_{R}$ contains $V$. The linear projection $\alpha: X_{3} \backslash X_{3} \cap V \rightarrow \mathbf{P}^{2}$ is dominant because the last assertion of the Second Claim implies that $\alpha$ does not contract infinitely many lines. Hence the linear projection of $X_{3}$ from the line $\langle\{A, B\}\rangle$ into $\mathbf{P}^{3}$ is a cone. By the Lemma proved in [6], Step 6 at p. $625, X_{3}$ is a cone, a contradiction.

Step 7. Here we will check that $\langle\{A, B\}\rangle$ is the only line containing $P$ and intersecting $X_{1} \backslash X_{1} \cap X_{2}$ and $X_{2} \backslash X_{1} \cap X_{2}$. Since the tangent developable of $X_{3}$ has dimension 4 and $P$ is general, $P$ is not contained in any line tangent to $X_{3}$ at one of its smooth points. Since $\left[X_{1} ; X_{2}\right]=\mathbf{P}^{5}$, the set $\mathcal{D}$ of all lines containing $P$ and intersecting both $X_{1} \backslash X_{1} \cap X_{2}$ and $X_{2} \backslash X_{1} \cap X_{2}$ is finite. Now we will check that $\mathcal{D}=\{\langle\{A, B\}\rangle\}$. Take any $D \in \mathcal{D}$. By the finiteness of $\mathcal{D}, D$ must be fixed as $\Pi$ varies. Hence $D \subseteq \Lambda_{\Pi} \cap \Lambda_{\Pi^{\prime}}=\langle\{A, B\}\rangle$ (Step 6).

Step 8. Call $\Gamma_{\Pi}$ the union of the one-dimensional components of $\Lambda_{\Pi} \cap X_{3}$. Here we will check that for general $\Pi$ the curve $\Gamma_{\Pi}$ is a line. Recall that $W_{P}$ is the closure of the union of all spaces $\Lambda_{\Pi}$ with $\Pi \in L_{P}$. Let $Y \subseteq \mathbf{P}^{r}$ be an irreducible $m$-dimensional variety, $m \geq 2$, containing a two-dimensional family of $(m-1)$ dimensional linear spaces. By [6], Lemma in Step 9 of $\S 2, Y$ is a linear space. Thus $W_{P}$ contains only a one-dimensional family of distinct 3 -spaces $\Lambda_{\Pi}$. Since $\operatorname{dim}\left(L_{P}\right)=3$ and each plane of $L_{P}$ belongs to some 3 -space $\Lambda_{\Pi}$ contained in $W_{P}$, it follows that the general plane $U$ of $\Lambda_{\Pi}$ containing $P$ intersects $X_{1}, X_{2}$ and $X_{3}$ and that, for general $P, \Pi$ and $U$, it intersects each $X_{i}$ exactly at one point (see 6], Cor. 1.3). Hence $\Gamma_{\Pi}$ is a line. Hence the variety $X_{3}$ contains an irreducible family $\Phi$ of lines $\Gamma_{\Pi}$, $\Pi$ general in $L_{P}$, with $\Gamma_{\Pi} \subset \Lambda_{\Pi}$. Since $\Lambda_{\Pi} \cap \Lambda_{\Pi^{\prime}}=\langle\{A, B\}\rangle$ for a general pair $\left(\Pi, \Pi^{\prime}\right)$ (Step 6$)$, we have $\operatorname{dim}(\Phi)>0$. Since $X_{3}$ is not a plane, we have $\operatorname{dim}(\Phi)=1$. If all lines $\Gamma_{\Pi}$ pass through a common point $Q$, then $X_{3}$ is a cone with vertex $Q$, contradicting our assumptions. Since not all lines $\Gamma_{\Pi}$ pass through a common point and $X_{3}$ is not a plane, we have $\Gamma_{\Pi} \cap \Gamma_{\Pi^{\prime}}=\emptyset$ for a general pair $\left(\Pi, \Pi^{\prime}\right)$ (Linear Lemma in [6], §1). We now give a side remark. Since $X_{3}$ is neither a plane nor a smooth quadric surface, $\Phi$ is the only positive-dimensional 
irreducible family of lines contained in $X_{3}$. Hence $\Phi$ does not depend on the choice of $P, A$ and $B$. For a general $B_{3} \in X_{3}$ there is a unique line $D\left(B_{3}\right)$ such that $B_{3} \in D\left(B_{3}\right) \subset X_{3}$. Since $\Phi$ covers $X_{3}$, we have $D\left(B_{3}\right) \in \Phi$. Notice that $D\left(B_{3}\right)$ depends only on $B_{3}$ and $X_{3}$, not on $X_{1}, X_{2}$ and the choices of $A, B$ and $P$ that we made to construct $\Phi$.

Step 9. Take a general triple $\left(A_{1}, A_{2}, A_{3}\right) \in X_{1} \times X_{2} \times X_{3}$. Hence $\left\langle\left\{A_{1}, A_{2}, A_{3}\right\}\right\rangle$ is a plane and a general element of $\operatorname{Sec}(\vec{X} ; 2, \vec{k})$, and hence $\left\langle\left\{A_{1}, A_{2}, A_{3}\right\}\right\rangle \cap X_{i}=$ $\left\{A_{i}\right\}$ (Lemma 21). A general $P \in\left\langle\left\{A_{1}, A_{2}, A_{3}\right\}\right\rangle$ may be considered as a general element of $\mathbf{P}^{5}$ because $\left[X_{1} ;\left[X_{2} ; X_{3}\right]\right]=\mathbf{P}^{5}$. Since $\left[X_{1} ; X_{2}\right]=\mathbf{P}^{5}$, there is $\left(B_{1}, B_{2}\right) \in X_{1} \times X_{2}$ such that $B_{1} \neq B_{2}$ and $P \in\left\langle\left\{B_{1}, B_{2}\right\}\right\rangle$; furthermore, there are only finitely many such pairs $\left(B_{1}, B_{2}\right)$. Conversely, given a general quadruple $\left(A_{1}^{\prime}, A_{2}^{\prime}, B_{1}^{\prime}, B_{2}^{\prime}\right) \in X_{1} \times X_{1} \times X_{2} \times X_{2}$, the 3 -dimensional linear space $\left\langle\left\{A_{1}, A_{2}, B_{1}, B_{2}\right\}\right\rangle$ intersects $X_{3}$ in a non-empty set and one point of this set, for fixed $A_{1}^{\prime}, A_{2}^{\prime}$ but for a general pair $\left(B_{1}^{\prime}, B_{2}^{\prime}\right)$, may be considered as a general point $A_{3}^{\prime}$ of $X_{3}$; furthermore, for general $A_{1}^{\prime}$ and $A_{2}^{\prime}$ we may find $A_{3}^{\prime}$ not collinear with $A_{1}^{\prime}$ and $A_{2}^{\prime}$. Hence $\left\langle\left\{A_{1}^{\prime}, A_{2}^{\prime}, A_{3}^{\prime}\right\}\right\rangle$ is a plane of $\left\langle\left\{A_{1}, A_{2}, B_{1}, B_{2}\right\}\right\rangle$, and thus it intersects the line $\left\langle\left\{B_{1}^{\prime}, B_{2}^{\prime}\right\}\right\rangle$. Since $\operatorname{dim}\left(\left\langle\left\{A_{1}, A_{2}, B_{1}, B_{2}\right\}\right\rangle\right)=3$, for general $B_{1}^{\prime}$ and $B_{2}^{\prime}$ we have $\left\langle\left\{A_{1}^{\prime}, A_{2}^{\prime}\right\}\right\rangle \cap\left\langle\left\{B_{1}^{\prime}, B_{2}^{\prime}\right\}\right\rangle=\emptyset$. Thus we may do the construction of Step 4 starting from $B_{1}^{\prime}, B_{2}^{\prime}$ and $P$ instead of $A, B$ and $P$. By construction $\left\langle\left\{A_{1}^{\prime}, A_{2}^{\prime}, A_{3}^{\prime}\right\}\right\rangle \in \Lambda_{P} \backslash \Lambda_{P^{\prime}}$. Thus by Step 8 the 3 -dimensional linear space $G$ spanned by $B_{1}^{\prime}, B_{2}^{\prime}$ and $\left\langle\left\{A_{1}^{\prime}, A_{2}^{\prime}, A_{3}^{\prime}\right\}\right\rangle$ intersects $X_{3}$ in a line $D\left(A_{1}^{\prime}, B_{1}^{\prime}, A_{2}^{\prime}, B_{2}^{\prime}\right) \in \Phi$. However, $G=\left\langle\left\{A_{1}^{\prime}, A_{2}^{\prime}, B_{1}^{\prime}, B_{2}^{\prime}\right\}\right\rangle$. Thus for a general quadruple $\left(A_{1}^{\prime}, A_{2}^{\prime}, B_{1}^{\prime}, B_{2}^{\prime}\right) \in X_{1} \times X_{1} \times X_{2} \times X_{2}$ the set $X_{3} \cap\left\langle\left\{A_{1}, A_{2}, B_{1}, B_{2}\right\}\right\rangle$ contains a line. Furthermore, $\left\langle\left\{A_{1}, A_{2}, B_{1}, B_{2}\right\}\right\rangle$ is spanned by $D\left(A_{1}^{\prime}, B_{1}^{\prime}, A_{2}^{\prime}, B_{2}^{\prime}\right)$, $A_{1}^{\prime}$ and $A_{2}^{\prime}$. Since $\operatorname{dim}(\Phi)=1$, we may find a one-dimensional irreducible family, $\Delta$, of pairs $\left(B_{1}^{\prime \prime}, B_{2}^{\prime \prime}\right) \in X_{1} \times X_{2}$ such that $X_{3} \cap\left\langle\left\{A_{1}, A_{2}, B_{1}, B_{2}\right\}\right\rangle=D\left(A_{1}^{\prime}, B_{1}^{\prime}, A_{2}^{\prime}, B_{2}^{\prime}\right)$ for every $\left(B_{1}^{\prime \prime}, B_{2}^{\prime \prime}\right) \in \Delta$. Hence $\left\langle\left\{A_{1}^{\prime}, A_{2}^{\prime}, B_{1}^{\prime}, B_{2}^{\prime}\right\}\right\rangle$ contains all such pairs $\left(B_{1}^{\prime \prime}, B_{2}^{\prime \prime}\right)$, contradicting Lemma 5 and hence concluding the proof.

Proof of Theorem Q If $X \subset \mathbf{P}^{m}, m \geq 5$, is a non-degenerate surface such that $\operatorname{dim}([X ; X])=4$, then $X$ is either a cone or a Veronese surface $([7])$. Notice that the role of the surface $X_{3}$ in the proof of Theorem 1 was quite different from the roles of $X_{1}$ and $X_{2}$, while the roles of $X_{1}$ and $X_{2}$ were exactly the same. The same proof works in the case $X_{1}=X_{2}$ (and proves Theorem 2), except that in Steps 4 and 9 we need to quote Lemma 3 instead of Lemma 2 and that in the proof of Theorem 1 we assumed and heavily used that $\operatorname{dim}\left(\left[X_{1} ; X_{2}\right]\right)=5$. Hence to complete the proof of Theorem 2 it is sufficient to check it when $\operatorname{dim}\left(\left[X_{1} ; X_{1}\right]\right) \leq 4$ and $X_{1}$ is not a cone, i.e. when $X_{1}$ is the Veronese surface. Assume $N=5$ and let $S \subset \mathbf{P}^{5}$ be the Veronese surface. Let $Y \subset \mathbf{P}^{5}$ be an integral non-degenerate surface with $Y \neq S$ and $Y$ not a cone. The pair $(S, Y)$ is not a $(1,(2,1))$-defective pair if and only if a general line $D \subset \mathbf{P}^{5}$ is contained in a plane spanned by two points of $S$ and one point of $Y$. Let $Z \subset \mathbf{P}^{5}$ be the secant variety of $S$. Thus $S$ is the hypersurface of $\mathbf{P}^{5}$ union all planes spanned by the conics contained in $S$. Let $D \subset \mathbf{P}^{5}$ be a general line. Fix $P \in D \cap Z$ and call $E \subset Z$ the plane such that $P \in E$ and $E \cap S=C$, where $C$ is a smooth conic. Set $M:=\langle E \cup D\rangle$. Thus $\operatorname{dim}(M)=3$. Take $Q \in M \cap Y$ and set $F:=E \cap\langle\{Q\} \cup D\rangle$. Hence $F$ is the intersection of two planes contained in $M$. The linear space $M$ moves if we move $D$ among the lines through $P$. For a general line $D$ containing $P$ the set $F$ is a line not tangent to $C$. 
Hence the general line $D$ containing $P$ is contained in the plane spanned by $Q$ and the two points of $F \cap C \subset S$, concluding the proof.

\section{FURTher RESUlts on $(t, \vec{k})$-DEFECTIVITY}

Let $\sigma: \mathbf{P}^{t} \times \mathbf{P}^{N} \rightarrow \mathbf{P}^{t N+t+N}$ be the Segre embedding. The proof of [8], Th. 2.1 (i.e. a computation of a certain Jacobian matrix), gives the following result.

Theorem 3. $\vec{X}$ is $(t, \vec{k})$-defective if and only if $\sigma\left(\mathbf{P}^{t} \times \vec{X}\right):=\left(\sigma\left(\mathbf{P}^{t} \times X_{1}\right), \ldots\right.$, $\left.\sigma\left(\mathbf{P}^{t} \times X_{s}\right)\right)$ is $\vec{k}$-defective and the total order of $(t, \vec{k})$-defectivity of $\vec{X}$ and the $\vec{k}$-defectivity of $\sigma\left(\mathbf{P}^{t} \times \vec{X}\right)$ are the same.

To obtain results for the $(t, \vec{k})$-defectivity it is essential to have results for the $\vec{k}$ defectivity of other joins; Theorem 3 is just one reason. Since the role of the varieties $X_{1}, \ldots, X_{s}$ is not the same if $k_{i} \neq k_{j}$ for some $i, j$ or the geometric properties (and even the dimensions) of the varieties $X_{1}, \ldots, X_{s}$ may be quite different (as, for instance, in Question (1), we introduce the following definition.

Definition 1. Fix $N, s, k_{i}, n_{i}, 1 \leq i \leq s$, and $t$ as above and assume that $\sum_{i=1}^{s} k_{i}\left(n_{i}+1\right) \leq N-1$. Fix an integer $i$ with $1 \leq i \leq s$ such that $k_{i}>0$. Set $\vec{k}(i):=\left(k_{1}^{\prime}, \ldots, k_{s}^{\prime}\right)$ with $k_{j}^{\prime}=k_{j}$ if $j \neq i$ and $k_{i}^{\prime}=k_{i}-1$. Set $\delta(\vec{X} ; \vec{k} ; i):=$ $\delta(\vec{X} ; \vec{k})-\delta(\vec{X} ; \vec{k}(i))$. The integer $\delta(\vec{X} ; \vec{k} ; i)$ will be called the $\vec{k}$-defect of $\vec{X}$ for the factor $i$.

The proof of 2], Th. 1.1, gives the following result.

Lemma 6. Fix $N, \vec{k}, i$ and $\vec{X}$ as above and assume that each variety $X_{j}$ is nondegenerate. Assume $\delta(\vec{X} ; \vec{k} ; i)>0$. Fix a general $P_{u, v} \in X_{u}, 1 \leq u \leq s$ and $1 \leq v \leq k_{u}$, and let $A$ be the linear span of the tangent spaces $\left(T X_{u}\right)_{P_{u, v}}, 1 \leq u \leq s$ and $1 \leq v \leq k_{u}$. Then the general hyperplane $H$ of $\mathbf{P}^{N}$ containing $A$ is tangent to $X_{i}$ at least along an irreducible variety of dimension $\delta(\vec{X} ; \vec{k} ; i)$ containing one of the points $P_{i, j}$ with $1 \leq j \leq k_{i}$.

Corollary 1. Fix $N, \vec{k}, i$ and $\vec{X}$ as above and assume that each variety $X_{j}$ is nondegenerate. Assume $\delta(\vec{X} ; \vec{k} ; i)>0$. Then $\operatorname{dim}\left(X_{i}\right)>\delta(\vec{X} ; \vec{k} ; i)$ and in particular $X_{i}$ is not a curve.

Remark 3. In Lemma 6 and Corollary 1 we may substitute the condition that each variety $X_{j}$ is non-degenerate with the condition that for each proper subspace $M$ of $\mathbf{P}^{N}$ containing some of the varieties $X_{j}$, say $X_{j}$ for $j \in S \subset\{1, \ldots, s\}$, we have $\sum_{j \in S} k_{j}\left(\operatorname{dim}\left(X_{j}\right)+1\right) \leq \operatorname{dim}(M)$.

Theorem 4. Fix an integer $s \geq 2$, positive integers $k_{1}, \ldots, k_{s}$ such that $k_{s}=1$ and integral subvarieties $X_{i} \subset \mathbf{P}^{N}$ such that $X_{j}$ is non-degenerate for every $j<s$ and $\operatorname{dim}\left(X_{s}\right)=1$. Set $\vec{k}:=\left(k_{1}, \ldots, k_{s}\right), \vec{k}(s):=\left(k_{1}, \ldots, k_{s-1}\right), \vec{X}:=\left(X_{1}, \ldots, X_{s}\right)$ and $\vec{X}(s):=\left(X_{1}, \ldots, X_{s}\right)$. Assume that $\vec{X}(s)$ is neither $\vec{k}(s)$-defective nor $(1, \vec{k}(s))$ defective and that $\operatorname{Sec}(\vec{X}(s) ; \vec{k}(s))$ is not a cone with vertex containing $X_{s}$. Then $\vec{X}$ is not $(1, \vec{k})$-defective.

Proof. Assume that $\vec{X}$ is $(1, \vec{k})$-defective. A general line $L \in \operatorname{Sec}(\vec{X} ; 1, \vec{k})$ is obtained by taking general $P_{i, j} \in X_{i}, 1 \leq i \leq s, 1 \leq j \leq k_{i}$, and then taking a general line $L$ contained in the $|\vec{k}|$-dimensional linear space spanned by the points $P_{i, j}$. Let 
$A(s)$ be the join of all points $P_{i, j}$ with $1 \leq i \leq s-1$ and $1 \leq j \leq k_{i}$. Set $Q(L):=A(s) \cap L$. Hence $Q(L) \in \operatorname{Sec}(\vec{X}(s) ; \vec{k}(s))$. By the generality of the points $P_{i, j}$ and of the line $L$ the point $Q(L)$ may be considered as a general point of $\operatorname{Sec}(\vec{X}(s) ; \vec{k}(s))$. By assumption there is a one-dimensional family of $|\vec{k}|$-planes, say $\left\{\Pi_{t}\right\}_{t \in T}$ with $T$ irreducible curve such that each $\Pi_{t}$ intersects each $X_{i}$ at $k_{i}$ distinct points, say $P_{i, j}(t), L \subset \Pi_{t}$ for every $t$, and there is $o \in T$ such that $P_{i, j}(o)=P_{i, j}$ for all $i, j$. Let $A(s, t)$ be the join of all points $P_{i, j}(t)$ with $1 \leq i \leq s-1$ and $1 \leq j \leq k_{i}$. Set $Q(L, t):=A(s, t) \cap L$. Hence $Q(L, t) \in A(s, t)$. First assume $Q(L, t)=Q(L)$ for every $t$. Since $Q(L, t):=A(s, t) \cap L$, this implies that $Q(L)$ is contained in infinitely many $(|\vec{k}|-1)$-dimensional linear spaces belonging to $\operatorname{Sec}(\vec{X}(s) ;|\vec{k}(s)|-1, \vec{k}(s))$. Since $Q(L)$ is general in $\operatorname{Sec}(\vec{X}(s) ; \vec{k}(s))$, this implies $\operatorname{dim}(\operatorname{Sec}(\vec{X}(s) ; \vec{k}(s)))) \leq \leq \sum_{i=1}^{s-1} k_{i}\left(\operatorname{dim}\left(X_{i}\right)+1\right)-2$, a contradiction. If $Q(L, t) \neq$ $Q(L)$ for general $t \in T$, then $L$ is contained in $\operatorname{Sec}(\vec{X}(s) ; \vec{k}(s))$. By the generality of $L$ we obtain $\operatorname{Sec}(\vec{X} ; \vec{k})=\operatorname{Sec}(\vec{X}(s) ; \vec{k}(s))$, i.e. $\operatorname{Sec}(\vec{X}(s) ; \vec{k}(s))$ is a cone with vertex containing $X_{s}$, a contradiction.

\section{REFERENCES}

[1] E. Ballico, Degenerate joins of surfaces in projective spaces, Int. Math. J. 2 (2002), 923-929. MR.1919681 (2003e:14044)

[2] E. Ballico, Weakly defective projective varieties, Int. Math. J. 2 (2002), 235-243. MR1913741 (2003e:14043)

[3] E. Ballico, Joins of projective varieties and flags of secant spaces, Int. J. Math. 3 (2003), no. 8, 843-846. MR,1990819 (2004f:14077)

[4] A. Calabri and C. Ciliberto, On special projections of varieties: epitome to a theorem of Beniamino Segre, Adv. Math. 1 (2001), 97-106. Mr 1823955(2002b:14064)

[5] L. Chiantini and C. Ciliberto, The Grassmannians of secant varieties of curves are not defective, Indag. Math., N. S., 13 (2002), no. 1, 23-28. MR.2014972 (2004h:14056)

[6] L. Chiantini and M. Coppens, Grassmannians of secant varieties, Forum Math. 13 (2001), 615-628. MR 1858491 (2002g:14079)

[7] M. Dale, Severi's theorem on the Veronese surface, J. London Math. Soc. 32 (1985), 419-425. MR 0825917 (87m:14043)

[8] C. Dionisi and C. Fontanari, Grassmann defectivity à la Terracini, Le Matematiche 56 (2001), no. 2, 245-255. Mr2009896 (2004h:14059)

[9] C. Fontanari, On Waring's problem for many forms and Grassmann defective varieties, J. Pure Appl. Algebra 174 (2002), no. 3, 243-247. MR.1929406|(2003h:14079)

Department of Mathematics, Univerity of Trento, 38050 Povo, Trento, Italy

E-mail address: ballico@science.unitn.it 